\title{
ENERGY THRESHOLD DETERMINATION FOR AMIGA MUON COUNTERS VIA GEANT4 SIMULATION
}

\author{
L. A. S. Pereira* \\ Unicamp, Instituto de Física 'Gleb Wataghin', 13083-859 Campinas SP, Brazil \\ E-mail: gutospeifi.unicamp.br
}

\section{A. C. Fauth}

Unicamp, Instituto de Física 'Gleb Wataghin', 13083-859 Campinas SP, Brazil

E-mail: fauth@ifi.unicamp.br

\begin{abstract}
Muons from extensive air shower with energy greater than or equivalent to $1 \mathrm{GeV}$ propagating in the soil are able to reach the AMIGA (Auger Muons and Infill for the Ground Array) detector. Although the air shower muonic component is attenuated much less than the electromagnetic component, the shielding of approximately $2.25 \mathrm{~m}$ of soil adds $540 \mathrm{~g} / \mathrm{cm}^{2}$ of vertical mass. Thus, in order to better understand attenuation mechanisms (shielding effects) of muons, a Monte Carlo simulation with Geant4 was made to determine the muon energy threshold. This threshold can be used to test the Geant4 simulation program, since the muon energy threshold is well calculated via the Bethe-Bloch formula. From the energy thresholds and the energy distribution at ground level for different particles from extensive air showers, the contribution of those particles to the data recorded by the detectors can be calculated. This contribution is crucial to correctly determine the number of muons in an extensive air shower, which is one of the main aims of the AMIGA enhancement.
\end{abstract}

The 34th International Cosmic Ray Conference,

30 July- 6 August, 2015

The Hague, The Netherlands

${ }^{*}$ Speaker. 


\section{Introduction}

One of the first improvements of the Pierre Auger Observatory is the AMIGA enhancement which aims the measurement of the cosmic ray spectrum and its chemical composition in the energy range from $10^{17} \mathrm{eV} \mathrm{[1].} \mathrm{One} \mathrm{of} \mathrm{its} \mathrm{main} \mathrm{objectives} \mathrm{is} \mathrm{to} \mathrm{lower} \mathrm{the} \mathrm{energy} \mathrm{threshold} \mathrm{for} \mathrm{full} \mathrm{trigger}$ efficiency to $10^{17} \mathrm{eV}$, so that the regions around the proposed second knee and the ankle in the energy spectrum of primary cosmic rays are fully covered by the Pierre Auger Observatory.

AMIGA consists of a surface detector array, which uses the same modular surface detectors of the Pierre Auger Observatory, but these are positioned in a hexagonal region with a distance of $750 \mathrm{~m}$ among the detectors and in a lower region the distance is of $433 \mathrm{~m} \mathrm{[2].}$

Muons with energy greater than or equivalent to $1 \mathrm{GeV}$ propagating in the soil are able to reach the muon detector. Although the air shower muonic component is attenuated much less than the electromagnetic component, the shielding of approximately $2.25 \mathrm{~m}$ of soil adds $540 \mathrm{~g} / \mathrm{cm}^{2}$ of vertical mass (approximately 60\% more than the atmosphere above the Pierre Auger Observatory) [1]. Thus, in order to better understand attenuation mechanisms (shielding effects) of muons and electrons, a Monte Carlo simulation with Geant4 was made to determine their energy threshold, i.e., the minimum kinetic energy they should have to go through the $2.25 \mathrm{~m}$ of soil and produce a signal in the AMIGA counters. The information on the energy threshold is important to understand the process of data analysis. This threshold can be used to test the Geant 4 simulation program, since the muon energy threshold is well calculated via the Bethe-Bloch formula.

\section{AMIGA counters}

The AMIGA counters count muons from extensive air showers observed by the Auger Observatory, which are reconstructed by Surface and Fluorescence detectors. They are composed of 30 $\mathrm{m}^{2}$ of buried detectors at a depth of $2.25 \mathrm{~m}$ corresponding to an atmospheric depth of $540 \mathrm{~g} / \mathrm{cm}^{2}$ [1]. $1 \mathrm{GeV}$ muons propagating in soil are able to reach the muon detectors which are located near the Cherenkov detector as shown in Figure 1.

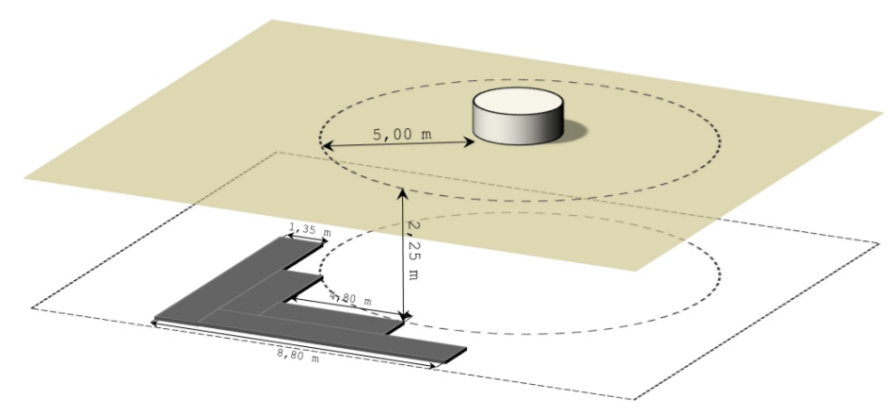

Figure 1: AMIGA detector pair with the muon counter (bottom) buried alongside a regular SD station (top). In this layout one muon counter module is replaced by two smaller modules with half the size thus the muon counter consists of four independent modules instead of three [3].

Each counter has three modules of $10 \mathrm{~m}^{2}$ sensitive area that are formed by strips of plastic scintillators with $4.1 \mathrm{~cm} \times 1.0 \mathrm{~cm}$ and $400 \mathrm{~cm}$ in length. Each module consists of 64 strips made 
of extruded polystyrene doped with fluor, POP (1) and POPOP (0.003) and co-extruded with a $\mathrm{Ti}_{2}$ reflective coating. The strips have a groove on the top side, in which a wavelength shifting optical fiber is glued and covered with a reflective foil. The fiber collector ends in a connector attached to a 64 multi-anode H7546B PMT manufactured by Hamamatsu. The scintillators are grouped into two sets of 32 strips on each side of the PMT [4].

The Geant 4 Monte Carlo simulation of the interaction of muons and electrons with the soil and counter is addressed in the following sections.

\section{Geant4 simulation}

Geant4 (Geometry and Tracking 4) Monte Carlo method is used to simulate the passage of particles through the soil and the counter where it is located and obtain information about the occurred interactions and produced particles, cross sections, mean free path, energy loss per unit lenght $(\mathrm{dE} / \mathrm{dx})$ and others.

\subsection{Geometry and Materials}

The Geant 4 is a platform which uses object-oriented programming in $\mathrm{C}++$. Based on this, it was initiated the simulation from a ready example and performed numerous code changes. These changes were made in order to build a simplified geometry of the AMIGA module counter in the soil. Among these changes there are the physical interactions for muons and electrons which occur through particle-matter interaction and determination of the energy loss $(\mathrm{dE} / \mathrm{dx})$ in the counter per event as well as the penetration depth reached by each simulated particle.

The geometry used in the simulation program is composed by: $i$ ) an outer volume, also called 'world volume', which defines the outer borders of the simulation. The dimensions of the world volume are $5 \mathrm{~m} \times 8 \mathrm{~m} \times 6 \mathrm{~m}$, centered around the origin of the rectangular coordinate system; ii) The soil volume, which has the dimensions of $5 \mathrm{~m} \times 8 \mathrm{~m} \times 3 \mathrm{~m}$ and is placed $1.5 \mathrm{~m}$ below the origin and finally iii) the muon counter module, which is placed at a depth of $2.25 \mathrm{~m}$. 


\subsection{Particle Primary Generator}

Set the characteristics of the building and the validation of the used models for the physics interaction processes, it was initiated the simulation by inciding particles over the top surface of the soil. The incidence of particles was based on a simulated cosmic ray shower with CORSIKA of a single, proton-induced extensive air shower at $10^{18.5} \mathrm{eV}$ as an input in Geant4 simulation (Figure 2).

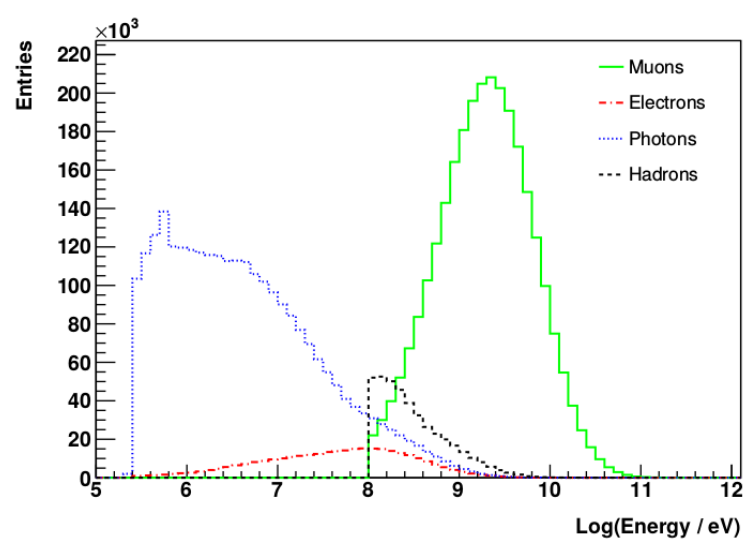

Figure 2: Energy distribution of different particle types on ground level from a single, proton-induced extensive air shower at $10^{18.5} \mathrm{eV} \mathrm{[5]} \mathrm{and} \mathrm{[6].}$

This shower has an applied energy cut for muons and hadrons at $100 \mathrm{MeV}$ and for electrons and photons at $0.25 \mathrm{MeV}$. Figure 2 shows the energy distributions of different particle types on ground level. Thus it was used the Geant4 reference class G4GeneralParticleSource (GPS) which allows to specify the spectral, spatial and angular distribution of the primary particle source. The source is a point beam centralized in the origin of the coordinate system over the surface of the Malargüe soil, being this electronic and muonic source vertically simulated.

The aim of this simulation is to determine the particle penetration depth and the energy threshold which are described in detail in the following sections.

\subsection{Determination of Energy Threshold}

The energy threshold is determined by taking into account the primary particle as well as the secondary particles produced in the soil above the detector. Moreover it can be understood as the minimum kinetic energy a particle originating from an extensive air shower needs in order to pass the $2.25 \mathrm{~m}$ of Malargüe soil and produce a signal in the muon counter. The information on the energy threshold is important to understand the process of data analysis. Furthermore it can be used to test the Geant4 simulation program, since the muon energy threshold is well calculated via the Bethe-Bloch formula [7].

The energy thresholds have been determined for muons and electrons since these are one of the most common particles from extensive air showers which reach the ground level. Later it is going to be studied the interaction as well as the energy threshold for other particles as photons, protons, neutrons and negatively charged pions.

The data from Geant 4 simulation has been analyzed using the ROOT toolkit, a $\mathrm{C}++$ based framework for data analysis [8]. 


\subsubsection{Muons}

For muon energy threshold determination, it was simulated 200000 vertical events over the top surface of the soil following the energy distribution for muons in Figure 2, which represents a Gaussian-like function distribution with mean at $1582 \mathrm{MeV}$. For each event, the penetration depth of the primary muon has been calculated using the $z$ coordinate of the endpoints of the respective muon track. Figure 3 shows the penetration depths of the primary muons.

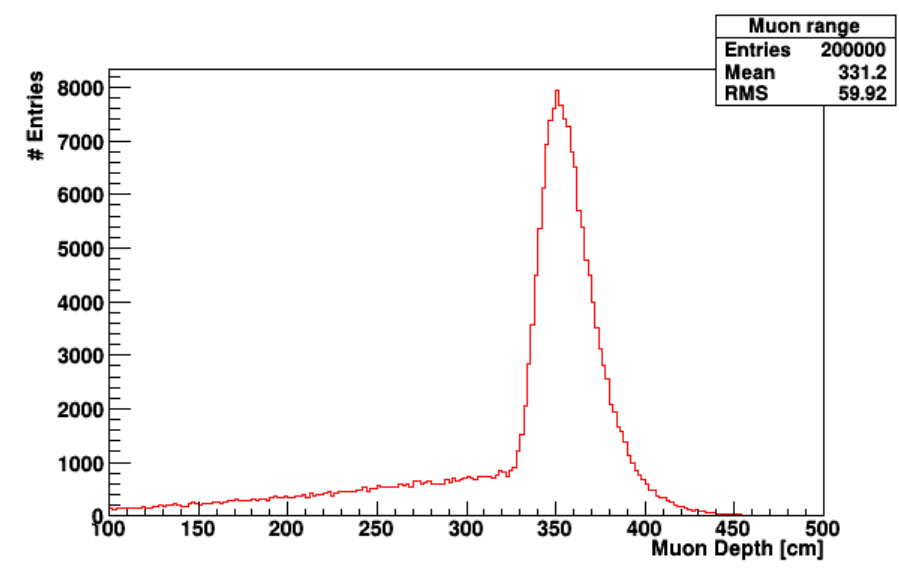

Figure 3: Penetration depths for muons following a Gaussian-like function incident energy distribution.

Through this distribution, the mean penetration depth in soil for muons with mean kinetic energy of $1574 \mathrm{MeV}$ is $\sim 331.2 \mathrm{~cm}$, implying they can reach the counter depositing an average energy at $\sim 2.088 \mathrm{MeV}$ as shown in Figure 4 . There is an amount of low energy muon particles which do not interact with the counter due to their decay before reaching the scintillator.
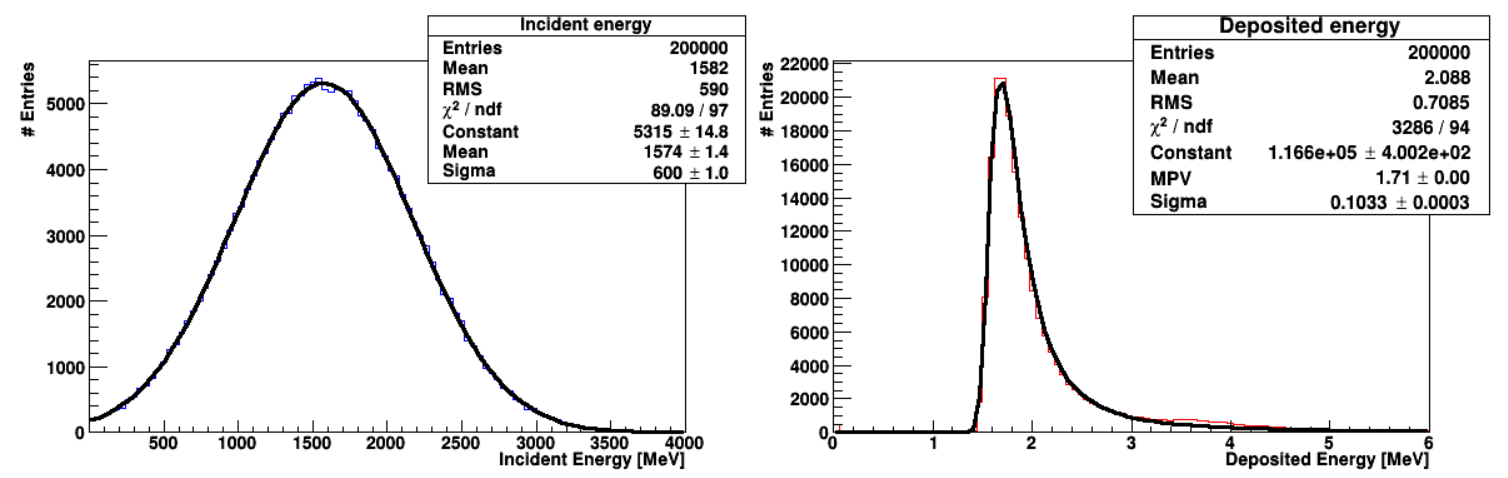

Figure 4: Muon incident energy distribution spectrum (left) and deposited energy in the counter by 200000 vertical muon events (right). The black curve in both spectra are the Gaussian (for the incident energy) and Landau (for the deposited energy) function fitting.

Muons with kinetic energy of $1582 \mathrm{MeV}$ can reach a penetration depth higher than the depth of the counter. Thus, to determine the energy threshold it was plotted the penetration depth against the kinetic energy of the primary muon, which provides a linear dependence, i. e., the higher the muon kinetic energy the higher the penetration depth is. Therefore a linear function of degree one was fitted and from the inverse function of the fitted curve the energy threshold was obtained. So 
for a penetration depth of $225 \mathrm{~cm}$, which corresponds to the counter position along the $z$ direction according to the simulation coordinate system, the minimun kinetic energy the muon should have to interact with the detector is at about $1074.73 \mathrm{MeV}$. This result greatly agrees with the CSDA range for muons in standard rock (see Figure 5) provided by NIST National Institute of Standards and Technology [9], in which the energy loss (stopping power) is calculated via Bethe-Block formula since the $\mathrm{dE} / \mathrm{dx}$ for muons in rock until energies $\sim 1.6 \mathrm{GeV}$ is through ionization and excitation process.
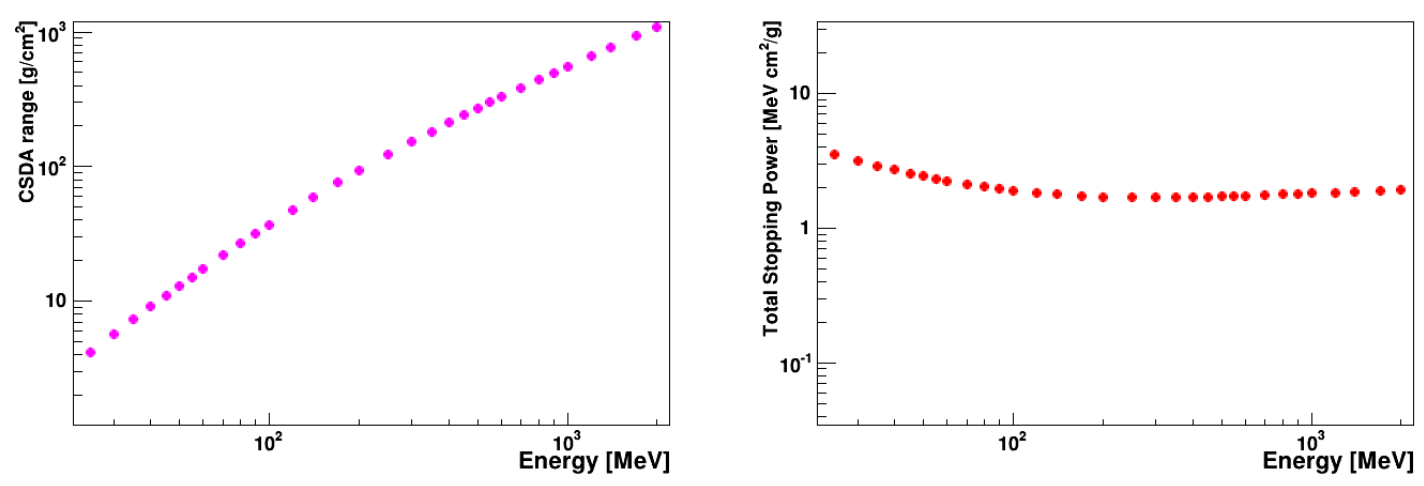

Figure 5: Muon interaction in standard rock with density of $2.65 \mathrm{~g} / \mathrm{cm}^{3}:$ muon CSDA in $\mathrm{g} / \mathrm{cm}^{2}$ (left) and muon stopping power in $\mathrm{MeV} \mathrm{cm} / \mathrm{g}$ (right) [9].

Unfortunately the NIST program does not provide a toolkit in which the target material can be constructed. The standard rock has a density of $2.65 \mathrm{~g} / \mathrm{cm}^{3}$ which is a bit higher than the density of Malarguie soil $\left(2.38 \mathrm{~g} / \mathrm{cm}^{3}\right)$ and therefore it provides an energy threshold a little higher at $225 \mathrm{~cm}$ depth.

Muons are the most particles from extensive air showers that reach the ground level. The remaining encompass electrons, photons and hadrons, mostly protons, neutrons and pions. These particles can interact with the soil above the detector and produce a signal in the AMIGA counter. Therefore, it is important to know and determine the energy thresholds for these particles in order to interpret the recorded data and better reconstruct the number of muons from the data. In the following section it is presented the electronic interaction and the energy threshold for this particle.

\subsubsection{Electrons}

Similarly for muon interaction, it was simulated 200000 electron events inciding vertically over the top surface of the soil with kinetic energy following the electronic energy distribution from Figure 2. This distribution can be approximated to Gaussian-like function with mean energy at $\sim 25.88 \mathrm{MeV}$. In Figure 6 the penetration depth along the $z$ direction in soil is shown. 


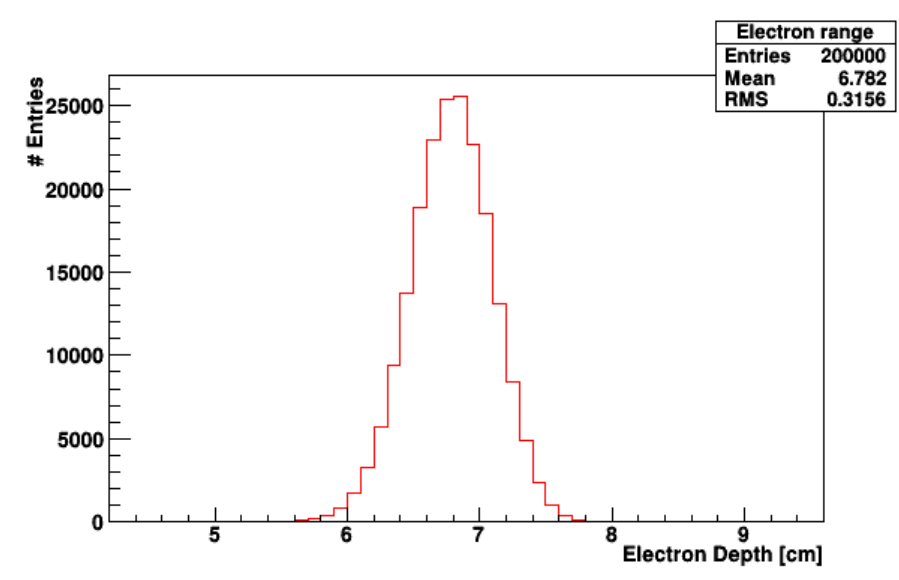

Figure 6: Penetration depths for electrons following a Gaussian-like function incident energy distribution.

The mean penetration depth for electrons is $6.782 \mathrm{~cm}$, which is far from the position where the counter is located not interacting with it. This result well agrees with the CSDA range distribution for electrons in Malargüe soil from EStar program [9], which calculates stopping power, density effect parameters, range, and radiation yield tables for electrons in various materials. In this program it is possible to create the desired material of interest. Figure 7 shows the CSDA for electrons with energy from $0.001 \mathrm{MeV}$ to $10000 \mathrm{MeV}$ in Malargüe soil and its stopping power.
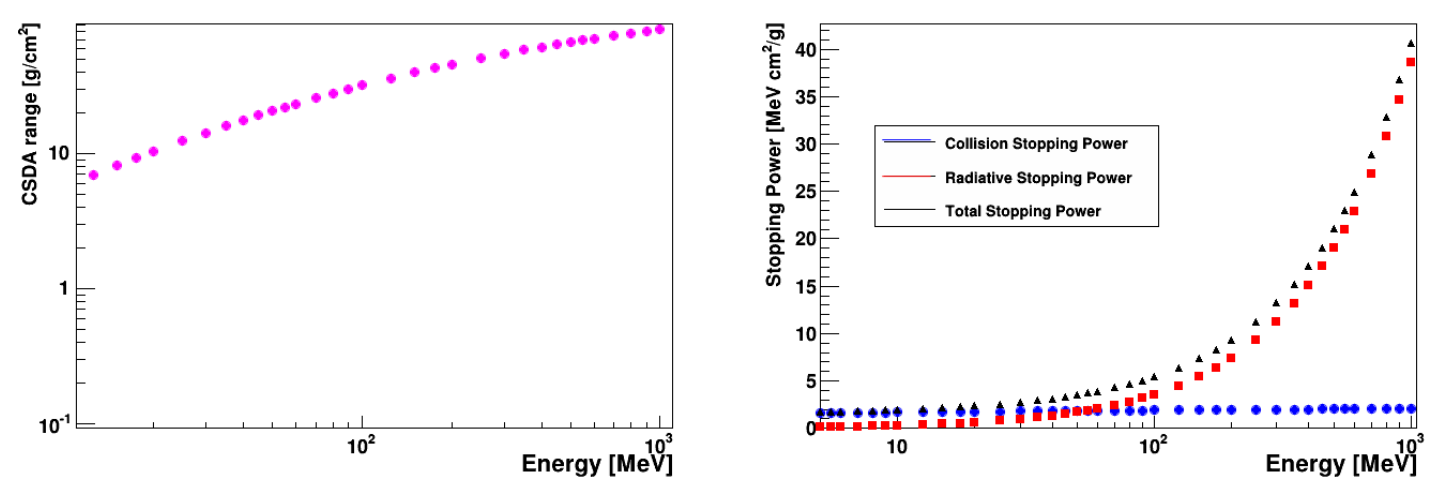

Figure 7: Electron interaction in Malargüe soil with density of $2.38 \mathrm{~g} / \mathrm{cm}^{3}$ : electronic CSDA in $\mathrm{g} / \mathrm{cm}^{2}$ (left) and electronic stopping power in $\mathrm{MeV} \mathrm{cm} / \mathrm{g}$ (right) [9]. 
According to the data for electrons interact with the AMIGA counters they should have an energy threshold of $\sim 858.6 \mathrm{MeV}$, which is $\sim 33$ times higher than the mean energy of electrons at ground level from extensive air showers. Thus, the soil completely shields the shower electronic component from interacting with the counter and produce a signal which can be misunderstood with a signal from a muon particle.

\section{Conclusion}

From the energy thresholds and the energy distribution at ground level for muons and electrons from extensive air showers, the contribution of those particles to the data recorded by the counters can be calculated. This contribution is crucial to better determine the number of muons in an extensive air shower, which is one of the main aims of the AMIGA enhancement. Moreover the calculated energy threshold could be validated comparing with the theoretical expectation, which energy loss (stopping power) for electrons and muons in soil is approximately described by the Bethe-Block formula since their main energy loss processes are by ionization and excitation. Moreover these thresholds can be used to optimize shower simulations (e.g. CORSIKA) to be more efficient in both computing time and disk space needed.

\section{Acknowledgments}

The authors would like to thank FAPESP (under grants No. 2013/23074-0) for the financial support.

\section{References}

[1] T. Waldenmaier, J. Blümer and H. Klages, Spectral resolved measurement of the nitrogen fluorescence emissions in air induced by electrons, Astroparticle Physics 29 (2008) 205-222.

[2] The AMIGA collaboration, AMIGA Design Report, Proposal for Evaluation by the Auger Assessment Committee (2006).

[3] U. Fröhlich, Charakterisierung der Szintillatoren und der Ausleseelektronik des AMIGA-Myon Systems, Masters thesis, University of Siegen, Germany (2009).

[4] F. Sánchez, Pierre Auger Collaboration, The AMIGA detector of the Pierre Auger Observatory: an overview, in proceedings of 32nd International Cosmic Rays Conference (2011).

[5] M. Settimo, Private communication, University of Siegen, Germany,(2011).

[6] M. Niechciol, Muon counter simulation studies for the AMIGA enhancement of the Pierre Auger Observatory, Master Thesis, University of Siegen, Germany (2011).

[7] H. A. Bethe, Zur Theorie des Durchgangs schneller Korpuskularstrahlen durch Materie, Annalen der Physik 5 (1930) 325-400.

[8] R. Brun, F. Rademakers, ROOT - An Object Oriented Data Analysis Framework, Nuclear Instrumentation Methods A 389 (1997) 81-86.

[9] M.J. Berger, J.S. Coursey, M.A. Zucker and J. Chang, ESTAR, PSTAR, and ASTAR: Computer Programs for Calculating Stopping-Power and Range Tables for Electrons, Protons, and Helium Ions (version 1.2.3), National Institute of Standards and Technology, Gaithersburg, MD (2005). 